\title{
SpS1-The Spitzer atlas of stellar spectra
}

\author{
David R. Ardila, W. Makowiecki, S. van Dyk, I. Song, J. Stauffer, \\ J. Rho, S. Fajardo-Acosta, D.W. Hoard and S. Wachter \\ ${ }^{1}$ Spitzer Science Center, California Institute of Technology, M/C 220-6, Pasadena, California \\ 91214, USA \\ email: dlp@ipac.caltech.edu
}

We present Spitzer Space Telescope spectra of 147 stars $(\mathrm{R} \sim 64-128, \lambda \lambda=5-35 \mu \mathrm{m}$, $\mathrm{S} / \mathrm{N}$ 100) covering most spectral and luminosity classes within the HR diagram. The spectra are available from the NASA/IPAC Infrared Science Archive (IRSA) and from the first author's webpage (http://web.ipac.caltech.edu/staff/ardila/Atlas/). The Atlas contains spectra of 'typical' stars, which may serve to refine galactic synthesis models, study stellar atmospheres, and establish a legacy for future IR missions, such as JWST.

The targets were selected from available public archival observations, complemented with a Spitzer cycle 5 DDT proposal (pid 485, PI Ardila). The priority was to include naked photospheres observed in the complete spectral range of the low-resolution Infrared Spectrograph (IRS). The presence of excess was determined by comparing the observed spectrum to an Engelke function (Engelke 1992). We have avoided objects described as young stars, RS CVn stars, Be stars, or spectroscopic binaries in the Simbad database. Spectral types were taken from the literature. We have included Wolf-Rayet and Emission-Line stars, as well as some stars of intrinsic interest (i.e. Cepheids, blue stragglers) independently of whether or not they present excess.

The spectra provided by IRS/SSC pipeline S18.7.0 were corrected for residual calibration errors, repeated observations were averaged, and the orders were matched. The error in the overall flux level is that of the standard IRS calibration $(\sim 5 \%)$. The spectra have not been corrected for interstellar extinction. In this Atlas, most stars from bright luminosity classes show interstellar silicate absorption at $10 \mu \mathrm{m}$ as well as reddened spectral slopes. For nearby (intrinsically faint) stars, the reddening correction is negligible.

The main sequence presents few spectral features over most of the temperature range. Lines from the Humphreys series of Hydrogen are present for some early A dwarfs. The fundamental $\mathrm{SiO}$ band $(7.55 \mu \mathrm{m})$ first becomes noticeable at $\mathrm{M} 0 \mathrm{~V}$ and remains strong until M6.5 V when it becomes confused with the $\mathrm{H}_{2} \mathrm{O}$ band at $6.27 \mu \mathrm{m}$. At later spectral classes, the spectra develop strong $\mathrm{CH}_{4}$ and $\mathrm{NH}_{3}$ bands (Cushing et al. 2006). The $\mathrm{SiO}$ band is strongly gravity dependent, and is observed as early as K0 for giants (also noted by Heras et al. 2002), remaining strong until M6 III, even while the water band starts developing (around M0 III).

This Atlas inherits a rich history of efforts in classifying the mid-IR spectra of stars. In particular, larger atlases based on IRAS (e.g. Kwok et al. 1997) and ISO (e.g. Kraemer et al. 2002) data are available. The primary goal of those atlases has been to establish an intrinsic IR classification scheme, one that does not rely on the optical spectral classification. The Spitzer Atlas, on the other hand, seeks to understand the spectral sequence at mid-IR wavelengths and as such it provides complementary information to those efforts.

\section{References}

Cushing, M. C. et al. 2006, ApJ, 648, 614

Heras, A. M. et al. 2002, A\&SA, 394, 539

Kraemer, K. E. et al. 2002, ApJS, 140, 389

Kwok, S. et al. 1997, ApJS, 112, 557 